\title{
Nonlinear optical properties of linear chain phosphazenes, $(\mathrm{PN})_{x}$
}

\author{
Prakash Chandra Jha \\ Solid State and Structural Chemistry Unit, Indian Institute of Science, Bangalore 560 012, India \\ Anu Krishnan and Puspendu K. Das \\ Department of Inorganic and Physical Chemistry, Indian Institute of Science, Bangalore 560 012, India \\ S. Ramasesha ${ }^{\text {a) }}$ \\ Solid State and Structural Chemistry Unit, Indian Institute of Science, Bangalore 560 012, India
}

\begin{abstract}
The correlated Pariser-Parr-Pople model Hamiltonian for interacting $\pi$-electrons is employed for calculating frequency dependent linear polarizability as well as first and second hyperpolarizabilities of linear chain phosphazenes $(-\mathrm{P}=\mathrm{N}-)_{x}(x=3-6)$. The model parameters for phosphorus and nitrogen are obtained by comparing the theoretical excitation energies with experimental spectra of the known phosphazene systems. The optical gap of the phosphazene oligomers extrapolates to 3.7 $\mathrm{eV}$ compared to $2.8 \mathrm{eV}$ of their organic analogs, namely, the polyenes. The linear polarizability of the phosphazene systems are comparable to those of the polyenes. However, the third harmonic generation coefficients are smaller at the same excitation energies. The power law exponent for the third harmonic generation coefficient in phosphazenes is also much smaller than that in polyenes. The second harmonic generation coefficients of the phosphazenes are smaller than those of the push-pull polyenes. Introduction of terminal push-pull groups on phosphazenes does not significantly improve the second harmonic generation response of these systems.
\end{abstract}

\section{INTRODUCTION}

Optical nonlinearities of conjugated organic systems have widely been studied in view of their potential application as active components in photonic and electro-optic devices. $^{1,2}$ The research in this area is focused on finding materials exhibiting large nonlinearity, fast response time, low transmission loss, and high thermal stability. In this context, the nonlinear optic (NLO) response of conjugated molecules that have been investigated theoretically and experimentally include linear chain polyenes, ${ }^{3-9}$ polynes, ${ }^{10,11}$ cumulenes, ${ }^{12}$ and cyanines. ${ }^{13,14}$ The results indicate that when donor and acceptor moieties are placed at the terminal positions of a conjugated backbone, both linear and nonlinear responses increase significantly. The magnitude of molecular polarizability and hyperpolarizability coefficients are found to increase superlinearly with an increase in the conjugation length (chain length of the backbone) between the donor and the acceptor. The strength of the donor and acceptor groups and order of their stacking along the backbone also play important roles in determining the magnitude of the response in various orders. The polyenes represent simple conjugated $\pi$-systems and the cyanine chain consists of a polyene chain with the terminal carbons replaced by nitrogen atoms. The number of electrons in the cyanine backbone is one more than the number of electrons in the polyacetylene chain of the same conjugation length. Several authors have reported measurements of the first order $(\beta)$ and second or$\operatorname{der}(\gamma)$ hyperpolarizabilities for polyenes with varying chain

\footnotetext{
${ }^{\text {a) }}$ Author to whom correspondence should be addressed. Electronic mail: ramasesh@sscu.iisc.ernet.in
}

lengths with donor and acceptor at the terminal positions. Blanchard-Desce et al. ${ }^{15}$ reported the weakest chain length dependence for $\beta$ in push-pull diphenylpolyenes at an excitation frequency of $0.65 \mathrm{eV}\left(\beta \sim L^{1.0}, L\right.$ is length). The exponent was found to vary significantly with the strength of donor and acceptor substituents. Huijts et al. ${ }^{16}$ measured a value of 3.4 for the exponent in diphenyl polyenes with stronger donor and acceptor groups at $1.167 \mathrm{eV}$. Albert et $a l .{ }^{17}$ have calculated the exponents for length dependencies of $\alpha, \beta$, and $\gamma$ in push-pull polyenes and they are 1.8 for $\alpha, 2.5$ for $\beta$, and 3.8 for $\gamma$ at an excitation energy of $1.167 \mathrm{eV}$ and for moderate push-pull strengths. Stevenson et al. ${ }^{13}$ measured the $\alpha$ and $\gamma$ values for cyanine dyes as a function of chain length at $0.65 \mathrm{eV}$ and observed an exponent of 1.0 for $\alpha$ and 7.8 for $\gamma$. Using the sum-over-states (SOS) formalism, Pierce ${ }^{14}$ reproduced the exponent of 8.0 for $\gamma$ of linear chain cyanines at $0.65 \mathrm{eV}$. Albert et al. ${ }^{17}$ have calculated the exponents as 3.04 and 4.0 for $\beta$ and $\gamma$ of cyanine dyes, respectively, at the same excitation energy using full configuration interaction studies of the Pariser-ParrPople (PPP) model Hamiltonian. ${ }^{18}$ It is apparent that the exponents obtained for the symmetric cyanine dyes are higher when compared to their polyene counterparts. ${ }^{12,13}$

The polyenes and linear phosphazenes have remarkable similarities arising from the existence of an in-plane interaction of nitrogen orbitals to form a conjugated pathway from nitrogen to the phosphorus. A large electronegativity difference between the phosphorus and the nitrogen atoms, makes the $\mathrm{P}-\mathrm{N}$ bond in linear phosphazenes highly polarizable. The polarizability of phosphazene chains can be further increased by placing donor and acceptor groups at the terminal posi- 


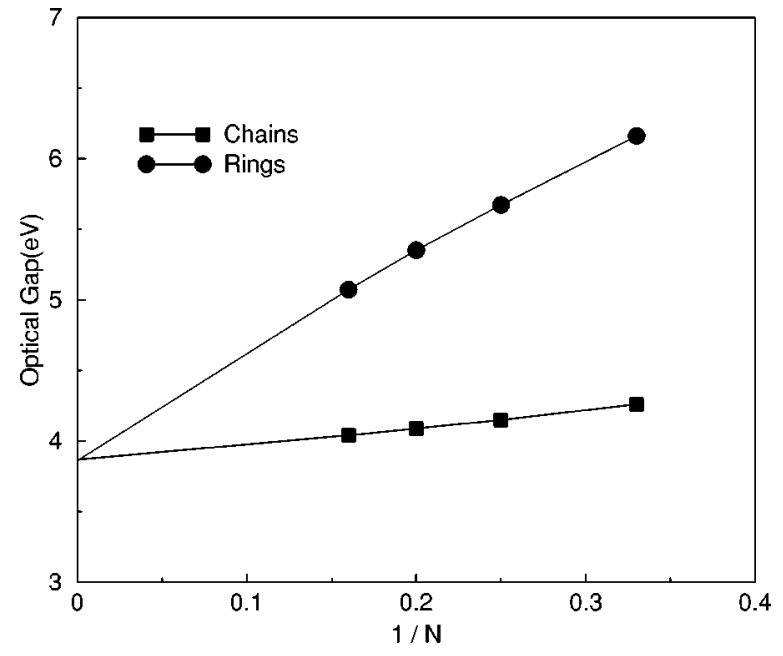

FIG. 1. Optical gap vs inverse system size for $(\mathrm{PN})_{x}$ systems.

tions. Previous reports on phosphazenes have discussed the participation of $d$-orbitals along with $p_{\pi}$-orbitals on nitrogen in bonding. Bonding in these systems can be qualitatively described as occurring through a $d_{\pi}-p_{\pi}$ delocalization. Paddock and co-workers ${ }^{19}$ have suggested that the nitrogen atom is $s p^{2}$ hybridized and the phosphorus atom is $s p^{3}$ hybridized in the $(-P=N-)_{x}$ chain. Out of the three $s p^{2}$ hybridized orbitals on the nitrogen atom two of them are utilized for $\sigma$-bonding with the substituents and the third has a pair of nonbonding electrons, thus leaving a lone electron in the unhybridized $p_{z}$ orbital available for $\pi$ bonding. On the other hand, $4 s p^{3}$ hybridized orbitals on the phosphorus atom are involved in $\sigma$ bonds, leaving a lone electron in the $d$ orbital for $\pi$ bonding. The $s p^{3}$ electron can now either interact with the lone electron on nitrogen through its $d_{x y}$ or $d_{x^{2}-y^{2}}$ orbitals to form an in plane $\pi$-bonded network. Dewar and co-workers ${ }^{20}$ have suggested the formation of a three center bond around each phosphorus. It has also been conjectured that the $\pi$-bonding is indeed a backbonding between the lone pair on the nitrogen and the $\sigma^{*}$ orbital of the $\mathrm{N}-\mathrm{P}$ bond. ${ }^{21}$

It is of interest to note that there exists no evidence either by experiments or theory to prove or disprove the above modes of bonding in linear $(\mathrm{PN})_{x}$ systems. However, what is clear is that each of the nitrogen and phosphorus atoms contribute one orbital towards the conjugation backbone. Based on the rudimentary picture of bonding, it is reasonable to assume that the number of electrons in the conjugated backbone equals the total number of nitrogen and phosphorus atoms forming the backbone. Thus, phosphazenes could be considered as the inorganic analogs of polyenes. This similarity has prompted our investigation of the linear and nonlinear optical properties of phosphazenes.

In this paper, we have used the PPP Hamiltonian and have calculated the exact dynamic linear and nonlinear optic coefficients of $(\mathrm{PN})_{x}$, where $x$ varies from 3 to 6 . Although a $\pi$-electron Hamiltonian is used explicitly in the calculations, the phosphorus $d$-orbital has been taken into consideration by the method of parameterization. This involves finding the values for phosphorus and nitrogen parameters to be used in

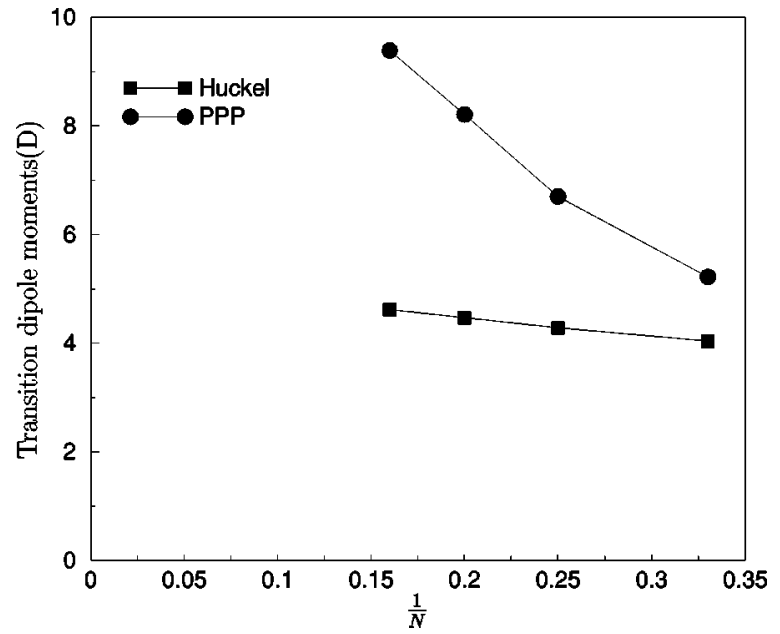

FIG. 2. Transition dipole moment for unsubstituted $(\mathrm{PN})_{x}$ chains vs inverse system size.

the PPP Hamiltonian. This is achieved by scanning a large set of reasonable parameters and choosing the set that best reproduces the experimental spectra known for similar phosphine-imine systems. Essentially the $d$-orbital contribution in the polarizability has been taken into consideration in a manner similar to that adopted for any semiempirical parameterization procedure. In this paper, the dynamic NLO coefficients $\alpha, \beta$, and $\gamma$ for the linear phosphazenes were calculated at excitation energies of 0.65 and $1.167 \mathrm{eV}$ by employing the correction vector $(\mathrm{CV})$ method and using a properly parametrized PPP Hamiltonian. The effect of pushpull groups on the dynamic optic response coefficients were also obtained. In the next section, we give a brief outline of the PPP model and the methodology employed by us. This will be followed by a section dealing with results and discussion.

\section{METHODOLOGY}

The PPP model Hamiltonian which takes into account explicit electron-electron interactions in a parametrized fashion has been discussed in detail in earlier papers, ${ }^{22,23}$ and only a brief presentation will be made here. The Hamiltonian in second-quantized notation is given by

$$
\begin{aligned}
& \hat{H}=\hat{H}_{0}+\hat{H}_{\mathrm{int}}, \\
& \hat{H}_{0}=\sum_{i} \epsilon_{i} \hat{n}_{i}+\sum_{\langle i j\rangle, \sigma} t_{i j}\left(\hat{a}_{i \sigma}^{\dagger} \hat{a}_{j \sigma}+\hat{a}_{j \sigma}^{\dagger} \hat{a}_{i \sigma}\right), \\
& \hat{H}_{\mathrm{int}}=\sum_{i} \frac{U_{i}}{2} \hat{n}_{i}\left(\hat{n}_{i}-1\right)+\sum_{i, j} V_{i j}\left(\hat{n}_{i}-z_{i}\right)\left(\hat{n}_{j}-z_{j}\right),
\end{aligned}
$$

where $\hat{a}_{i \sigma}^{\dagger}\left(\hat{a}_{i \sigma}\right)$ creates (annihilates) an electron with spin $\sigma$ in the orbital involved in conjugation at site $i, \epsilon_{i}$ is the orbital energy of the orbital involved in conjugation at site $i, t_{i j}$, the transfer integral or the Hückel resonance integral between the bonded sites $i$ and $j$, and $U_{i}$ is the on-site correlation energy of the orbital at site $i$. The interorbital or intersite electron repulsion parameter $V_{i j}$ is given by the Ohno parameterization, ${ }^{24}$ 
TABLE I. The different components of the linear polarizability $\alpha$ (in a.u.) at two different excitation frequencies 0.65 and $1.167 \mathrm{eV}$ for phosphazenes. Values in parentheses correspond to polyenes and are presented for comparison ( 1 a.u. $\left.=1.48 \times 10^{-23} \mathrm{esu}\right)$.

\begin{tabular}{clccc}
\hline \hline $\mathrm{N}$ & $\hbar \omega(\mathrm{eV})$ & $\alpha_{x x}$ & $\alpha_{x y}$ & $\alpha_{y y}$ \\
\hline \multirow{2}{*}{6} & 0.65 & $84.07(85.33)$ & $35.93(25.78)$ & $19.15(13.48)$ \\
& 1.167 & $87.96(88.62)$ & $37.55(26.73)$ & $18.68(13.82)$ \\
8 & 0.65 & $125.28(139.5)$ & $50.67(36.75)$ & $26.44(18.48)$ \\
& 1.167 & $87.96(88.62)$ & $37.55(26.73)$ & $18.68(13.82)$ \\
10 & 0.65 & $168.9(201.8)$ & $65.61(48.51)$ & $33.76(23.60)$ \\
& 1.167 & $178.2(212.9)$ & $69.12(51.00)$ & $35.22(24.32)$ \\
12 & 0.65 & $214.3(270.2)$ & $80.62(60.86)$ & $41.07(28.81)$ \\
& 1.167 & $226.6(286.9)$ & $85.15(64.36)$ & $42.90(29.76)$ \\
\hline \hline
\end{tabular}

$$
V_{i j}=14.397\left[\left(\frac{28.794}{U_{i}+U_{j}}\right)^{2}+r_{i, j}^{2}\right]^{-(1 / 2)},
$$

where the distances $R_{i j}$ are in $\AA$, the energies $U_{i}, V_{i j}$ are in $\mathrm{eV}$ and $z_{i}$ is the local chemical potential of the orbital given by the number of electrons contributed by the orbital $i$ to the conjugation backbone. While all these parameters are well parametrized for carbon and nitrogen, for other atoms they have not been determined. Parameterization was carried out for nitrogen ${ }^{17}$ while treating the cyanine dyes in the past. In the cyanine dyes, the terminal nitrogen atoms contribute a pair of electrons to conjugation and their parameters have been optimized to site energy, $\epsilon_{N}=-18.43 \mathrm{eV}$ and on-site correlation energy, $U_{N}=15 \mathrm{eV}$ relative to the carbon values of 0.0 and $11.26 \mathrm{eV}$, respectively. ${ }^{22}$ The larger $U_{N}$ value reflects the more compact $p$-orbital in nitrogen and the large negative value of $\epsilon_{N}$ leads to near double occupancy of the nitrogen orbital. The nitrogen parameters when its orbital involved in conjugation is contributing just one electron has been optimized to $\epsilon_{N}=-2.96 \mathrm{eV}$ and $U_{N}=12.34 \mathrm{eV}$, once again relative to carbon parameters. the accepted value of the transfer integral for the $\mathrm{C}-\mathrm{C}$ bond for a bond distance of $1.397 \AA$ is $2.4 \mathrm{eV}$. The nitrogen parameters in phosphazenes will be different as they will be determined relative to phosphorus than to carbon as $(\mathrm{PN})_{x}$ does not have any carbon atoms in the conjugation path.

The PPP Hamiltonian considers only one active orbital per site, namely, the $\pi$ orbitals on the site. This approximation is valid when the $\sigma$ and $\pi$ excitation are well separated energetically. This appears to be the case in long conjugated oligomers. Restricting to only one orbital per site has the advantage that the full configuration interaction $(\mathrm{CI})$ space is large but manageable even for fairly large molecules. The Hamiltonian matrix in this space is also rather sparse and hence routinely allows accessing several low-lying states for full CI space of a few million configurations. For a given molecule, an $a b$ initio calculation within a restricted CI space gives quite reliable excitation spectrum. However, while studying oligomers of different sizes to obtain information of the excitation spectrum in the polymer limit, the restricted CI $a b$ initio calculations have the disadvantage of not preserving size consistency. In this regard, modeling polymers using a properly parametrized PPP model within full CI scheme is known to lead to proper description of the polymer limit. ${ }^{25}$

Various theoretical methods used for calculating the nonlinear coefficients include the finite field method (FFM) ${ }^{26}$ the sum-over-states method (SOS), ${ }^{27}$ and the CV method. ${ }^{28}$ In the FFM method, an external electric field is applied and the associated change in the total energy or dipole moment are computed. From these changes obtained at various field strengths, the linear and nonlinear optic coefficients are calculated as appropriate derivatives. The NLO coefficients thus obtained are static while other methods can yield dynamic coefficients. In the SOS method, energies of several (about 30 or more) low-lying excited states and transition moments among these states are computed for the Hamiltonian of the system. Using these quantities, explicit perturbation summations for each coefficient are performed at desired frequencies. In this method the number of excited states retained is arbitrary. The assumption is that the sums are well behaved and convergence would be achieved with as few as 10-30 low-lying excited states even though the dimension of the configuration space is much larger (in millions or more).

TABLE II. The different components of the linear polarizability $\alpha$ (in a.u., 1 a.u. $=1.48 \times 10^{-23} \mathrm{esu}$ ) at two different frequencies 0.65 and $1.167 \mathrm{eV}$ for push-pull phosphazenes. Values in parentheses correspond to push-pull polyenes and are presented for comparison.

\begin{tabular}{clccc}
\hline \hline $\mathrm{N}$ & $\hbar \omega(\mathrm{eV})$ & $\alpha_{x x}$ & $\alpha_{x y}$ & $\alpha_{y y}$ \\
\hline \multirow{2}{*}{6} & 0.65 & $81.64(87.72)$ & $33.90(26.30)$ & $18.03(13.73)$ \\
& 1.167 & $85.55(91.22)$ & $35.41(27.30)$ & $18.68(14.09)$ \\
8 & 0.65 & $122.2(142.7)$ & $49.03(37.33)$ & $25.49(18.73)$ \\
& 1.167 & $128.5(149.6)$ & $51.46(39.04)$ & $26.52(19.28)$ \\
10 & 0.65 & $165.0(205.6)$ & $64.22(49.14)$ & $32.93(23.86)$ \\
& 1.167 & $174.1(217.2)$ & $67.63(51.72)$ & $34.33(24.61)$ \\
12 & 0.65 & $209.7(274.5)$ & $79.39(61.53)$ & $40.31(29.07)$ \\
& 1.167 & $221.8(291.8)$ & $83.82(65.12)$ & $42.09(30.04)$ \\
\hline \hline
\end{tabular}


TABLE III. The different components of the first hyperpolarizability $\beta$ (in a.u, 1 a.u. $=8.637 \times 10^{-32}$ esu) for various linear $(\mathrm{PN})_{x}$ systems, at $\epsilon_{\mathrm{P}}=0.0$ eV and $\epsilon_{\mathrm{N}}=-5.8 \mathrm{eV}$.

\begin{tabular}{|c|c|c|c|c|c|c|c|}
\hline $\mathrm{N}$ & $\hbar \omega(\mathrm{eV})$ & $\beta_{x x x}$ & $\beta_{x x y}$ & $\beta_{x y y}$ & $\beta_{y x x}$ & $\beta_{y x y}$ & $\beta_{y y y}$ \\
\hline \multirow[t]{2}{*}{6} & 0.65 & 2.43 & 3.98 & 6.03 & 2.91 & 5.72 & 3.46 \\
\hline & 1.167 & -20.50 & 4.19 & 8.27 & -2.07 & 6.65 & 4.58 \\
\hline \multirow[t]{2}{*}{8} & 0.65 & 29.80 & -14.00 & -0.24 & -14.17 & -0.66 & 1.44 \\
\hline & 1.167 & -0.88 & -21.07 & -0.19 & -24.11 & -2.47 & 1.82 \\
\hline \multirow[t]{2}{*}{10} & 0.65 & 94.26 & -39.67 & -10.38 & -37.44 & -10.79 & -1.93 \\
\hline & 1.167 & 77.49 & -57.31 & -14.62 & -49.03 & -16.90 & -3.04 \\
\hline \multirow[t]{2}{*}{12} & 0.65 & 190.7 & -70.58 & -23.11 & -64.67 & -23.40 & -6.17 \\
\hline & 1.167 & -209.6 & 101.2 & 33.28 & 74.30 & 34.80 & 9.30 \\
\hline
\end{tabular}

However, such summations lead to uncontrolled errors in the optic coefficients. ${ }^{7}$ Morley et al. ${ }^{8}$ have achieved convergence within the first 50 states of polyenes. On the other hand, Docherty and co-workers ${ }^{29}$ have shown that convergence could not be achieved in the case of 4,4'-N,N-dimethyl amino nitrostilbene. While in the SOS scheme, it can be claimed that the parametrization of the model Hamiltonian somehow includes the effects of higher order excitations and therefore it is inappropriate to include higher excitations, there is no convincing physical basis for this argument and it is largely an article of faith.

In order to extrapolate the NLO properties of oligomers of different sizes to the polymer limit and also to obtain information such as system size dependence of the NLO coefficients, it is necessary to compute model exact or full CI NLO properties of the system as described by the model Hamiltonian. Full CI NLO properties can indeed be obtained by employing the correction vector $(\mathrm{CV})$ technique. The $\mathrm{CV}$ method, being equivalent to a full SOS method in the chosen model space, also completely avoids the problem of convergence inherent in the truncated SOS method. In the CV method, without resorting to explicitly solving for the excited states and the corresponding transition dipoles, we obtain the NLO coefficients from the correction vectors which are obtained by solving inhomogeneous linear algebraic equations. It involves solving for the vectors $\phi_{i}^{(1)}\left(\omega_{1}\right)$ and $\phi_{i j}^{(2)}\left(\omega_{1}, \omega_{2}\right)$ which are defined by

$$
\begin{aligned}
& \left(\mathbf{H}-E_{G}+\hbar \omega_{1}+i \Gamma\right) \phi_{i}^{(1)}\left(\omega_{1}\right)=\tilde{\mu}_{i}|G\rangle, \\
& \left(\mathbf{H}-E_{G}+\hbar \omega_{2}+i \Gamma\right) \phi_{i j}^{(2)}\left(\omega_{1}, \omega_{2}\right)=\tilde{\mu}_{j} \phi_{i}^{(1)}\left(\omega_{1}\right),
\end{aligned}
$$

where $\mathbf{H}$ is the Hamiltonian matrix in the chosen many-body basis, $E_{G}$ is the ground state energy, $\omega_{1}, \omega_{2}$ are the frequencies, and $\hat{\mu}_{i}$ is the $i$ th component of the dipole displacement operator, $\tilde{\mu}_{i}=\widehat{\mu_{i}}-\left\langle G\left|\widehat{\mu_{i}}\right| G\right\rangle$ and $\Gamma$ is the average lifetime of the excited states. It can be shown that $\phi_{i}^{(1)}\left(\omega_{1}\right)$ and $\phi_{i j}^{(2)}\left(\omega_{1}, \omega_{2}\right)$ expressed in the basis of the eigenstates of the Hamiltonian $|R\rangle$ are given by

$$
\phi_{i}^{(1)}\left(\omega_{1}\right)=\sum_{R} \frac{\left\langle R\left|\tilde{\mu}_{i}\right| G\right\rangle}{E_{R}-E_{G}+\hbar \omega_{1}+i \Gamma}|R\rangle
$$

\begin{tabular}{|c|c|c|c|c|c|c|c|}
\hline $\mathrm{N}$ & $\hbar \omega(\mathrm{eV})$ & $\beta_{x x x}$ & $\beta_{x x y}$ & $\beta_{x y y}$ & $\beta_{y x x}$ & $\beta_{y x y}$ & $\beta_{y y y}$ \\
\hline \multirow[t]{2}{*}{6} & 0.65 & $\begin{array}{c}-29.08 \\
(-354.73)\end{array}$ & $\begin{array}{c}37.17 \\
(-69.74)\end{array}$ & $\begin{array}{c}21.25 \\
(-9.59)\end{array}$ & $\begin{array}{c}33.51 \\
(-75.95)\end{array}$ & $\begin{array}{c}20.76 \\
(-11.48)\end{array}$ & $\begin{array}{c}9.98 \\
(0.05)\end{array}$ \\
\hline & 1.167 & $\begin{array}{c}-94.12 \\
(-532.17)\end{array}$ & $\begin{array}{c}46.65 \\
(-97.74)\end{array}$ & $\begin{array}{c}28.69 \\
(-10.19)\end{array}$ & $\begin{array}{r}25.63 \\
(-127.72)\end{array}$ & $\begin{array}{c}25.92 \\
(-19.32)\end{array}$ & $\begin{array}{c}13.02 \\
(0.07)\end{array}$ \\
\hline \multirow[t]{2}{*}{8} & 0.65 & $\begin{array}{c}-20.45 \\
(-748.70)\end{array}$ & $\begin{array}{r}24.81 \\
(-164.90)\end{array}$ & $\begin{array}{c}18.72 \\
(-27.34)\end{array}$ & $\begin{array}{r}21.04 \\
(-178.11)\end{array}$ & $\begin{array}{r}18.00 \\
(-31.69)\end{array}$ & $\begin{array}{r}9.54 \\
(-1.53)\end{array}$ \\
\hline & 1.167 & $\begin{array}{c}-113.1 \\
(-1118.86)\end{array}$ & $\begin{array}{r}27.62 \\
(-231.82)\end{array}$ & $\begin{array}{c}25.49 \\
(-31.21)\end{array}$ & $\begin{array}{r}3.95 \\
(-295.42)\end{array}$ & $\begin{array}{c}21.37 \\
(-52.18)\end{array}$ & $\begin{array}{c}12.53 \\
(-2.29)\end{array}$ \\
\hline \multirow[t]{2}{*}{10} & 0.65 & $\begin{array}{r}36.01 \\
(-1148.72)\end{array}$ & $\begin{array}{r}2.72 \\
(-275.14)\end{array}$ & $\begin{array}{c}10.62 \\
(-52.94)\end{array}$ & $\begin{array}{c}0.84 \\
(-294.23)\end{array}$ & $\begin{array}{r}9.84 \\
(-59.54)\end{array}$ & $\begin{array}{r}6.99 \\
(-6.00)\end{array}$ \\
\hline & 1.167 & $\begin{array}{c}-46.92 \\
(-1690.70)\end{array}$ & $\begin{array}{c}-4.28 \\
(-384.13)\end{array}$ & $\begin{array}{c}13.97 \\
(-63.52)\end{array}$ & $\begin{array}{c}-19.06 \\
(-474.71)\end{array}$ & $\begin{array}{c}9.47 \\
(-94.83)\end{array}$ & $\begin{array}{r}8.88 \\
(-8.83)\end{array}$ \\
\hline 12 & $\begin{array}{l}0.65 \\
1.167\end{array}$ & $\begin{array}{c}-126.6 \\
(-1297.01) \\
-77.79 \\
(-1896.03)\end{array}$ & $\begin{array}{r}25.40 \\
(-331.50) \\
44.19 \\
(-464.04)\end{array}$ & $\begin{array}{c}0.9088 \\
(-73.08) \\
2.85 \\
(-92.43)\end{array}$ & $\begin{array}{c}23.96 \\
(-350.48) \\
42.03 \\
(-553.46)\end{array}$ & $\begin{array}{r}1.61 \\
(-79.73) \\
6.90 \\
(-123.76)\end{array}$ & $\begin{array}{c}-3.18 \\
(-12.85) \\
-3.27 \\
(-18.78)\end{array}$ \\
\hline
\end{tabular}

TABLE IV. The SHG coefficients (in a.u., 1 a.u. $=8.637 \times 10^{-32} \mathrm{esu}$ ) of the substituted linear $(\mathrm{PN})_{x}$ system at excitation frequencies 0.65 and $1.167 \mathrm{eV}$. The values in parentheses are obtained from a two state model. 
TABLE V. The SHG coefficients (in a.u., 1 a.u. $=8.637 \times 10^{-32}$ esu) of the substituted linear $\mathrm{CH}_{X}$ system at excitation frequencies 0.65 and 1.167 eV. The values in parentheses are from a two state model.

\begin{tabular}{|c|c|c|c|c|c|c|c|}
\hline $\mathrm{N}$ & $\hbar \omega(\mathrm{eV})$ & $\beta_{x x x}$ & $\beta_{x x y}$ & $\beta_{x y y}$ & $\beta_{y x x}$ & $\beta_{y x y}$ & $\beta_{y y y}$ \\
\hline \multirow[t]{4}{*}{6} & \multirow[t]{2}{*}{0.65} & 106.60 & 10.19 & 7.14 & 10.93 & 7.10 & 2.63 \\
\hline & & $(209.42)$ & $(40.64)$ & $(6.45)$ & $(42.37)$ & (6.93) & $(0.59)$ \\
\hline & \multirow[t]{2}{*}{1.167} & 140.61 & 14.50 & 9.38 & 17.60 & 9.25 & 3.49 \\
\hline & & $(260.64)$ & $(49.00)$ & (7.15) & $(55.93)$ & (9.07) & $(0.73)$ \\
\hline \multirow[t]{4}{*}{8} & \multirow[t]{2}{*}{0.65} & 221.75 & 15.26 & 10.30 & 17.20 & 10.25 & 3.45 \\
\hline & & $(409.92)$ & $(74.72)$ & $(12.31)$ & $(77.50)$ & $(12.98)$ & (1.78) \\
\hline & \multirow[t]{2}{*}{1.167} & 315.35 & 22.97 & 14.19 & 32.00 & 14.14 & 4.83 \\
\hline & & $(539.79)$ & (95.68) & (14.92) & (107.48) & (17.74) & $(2.35)$ \\
\hline \multirow[t]{4}{*}{10} & \multirow[t]{2}{*}{0.65} & 369.05 & 20.72 & 13.06 & 24.42 & 13.03 & 4.12 \\
\hline & & $(681.28)$ & (112.68) & (16.97) & (117.33) & (17.97) & $(2.30)$ \\
\hline & \multirow[t]{2}{*}{1.167} & 564.50 & 32.24 & 18.73 & 51.25 & 19.03 & 6.07 \\
\hline & & $(945.15)$ & (151.54) & (21.49) & (172.34) & (25.96) & $(3.20)$ \\
\hline \multirow[t]{4}{*}{12} & \multirow[t]{2}{*}{0.65} & 533.01 & 26.47 & 15.39 & 32.31 & 15.37 & 4.66 \\
\hline & & (1001.12) & (151.54) & $(20.64)$ & (151.88) & $(22.10)$ & $(2.50)$ \\
\hline & \multirow[t]{2}{*}{1.167} & 872.81 & 41.83 & 22.71 & 74.70 & 23.67 & 7.18 \\
\hline & & (1456.44) & $(212.55)$ & $(26.87)$ & (246.97) & (33.73) & (3.63) \\
\hline
\end{tabular}

$\phi_{i j}^{(2)}\left(\omega_{1}, \omega_{2}\right)=\sum_{S} \sum_{R} \frac{\left\langle S\left|\tilde{\mu}_{j}\right| R\right\rangle\left\langle R\left|\tilde{\mu}_{i}\right| G\right\rangle}{\left(E_{R}-E_{G}+\hbar \omega_{1}+i \Gamma\right)\left(E_{S}-E_{G}+\hbar \omega_{2}+i \Gamma\right)}|S\rangle$.

Therefore the $\phi_{i}^{(1)}\left(\omega_{1}\right)$ and $\phi_{i j}^{(2)}\left(\omega_{1}, \omega_{2}\right)$ can be readily used to compute linear and nonlinear frequency dependent polarizabilities within the PPP model. The NLO coefficients in terms of these correction vectors are given by

$$
\begin{aligned}
& \alpha_{i j}(\omega)=\left(\left\langle\phi_{i}^{(1)}(\omega)\left|\widehat{\mu_{j}}\right| G\right\rangle+\left\langle\phi_{i}^{(1)}(-\omega)\left|\widehat{\mu_{j}}\right| G\right\rangle\right), \\
& \beta_{i j k}\left(\omega_{1}, \omega_{2}\right)=\hat{P}_{i j k}\left(\left\langle\phi_{i}^{(1)}\left(-\omega_{1}-\omega_{2}\right)\left|\widehat{\mu_{j}}\right| \phi_{k}^{(1)}\left(-\omega_{2}\right)\right\rangle\right), \\
& \gamma_{i j k l}\left(\omega_{1}, \omega_{2}, \omega_{3}\right)= \hat{P}_{i j k l}\left(\left\langle\phi_{i}^{(1)}\left(-\omega_{1}-\omega_{2}-\omega_{3}\right)\right|\right. \\
&\left.\times \widehat{\mu_{j}}\left|\phi_{k l}^{(2)}\left(-\omega_{1}-\omega_{2},-\omega_{1}\right)\right\rangle\right),
\end{aligned}
$$

where the operators $\hat{P}_{i j k}$ and $\hat{P}_{i j k l}$ generate all permutations: $\left(-\omega_{\sigma}, i\right),\left(\omega_{1}, j\right),\left(\omega_{2}, k\right)$ and $\left(-\omega_{\sigma}, i\right),\left(\omega_{1}, j\right),\left(\omega_{2}, k\right)$, $\left(\omega_{3}, l\right)$ leading to six terms for $\beta$ (with $\omega_{\sigma}=\omega_{1}+\omega_{2}$ ) and 24

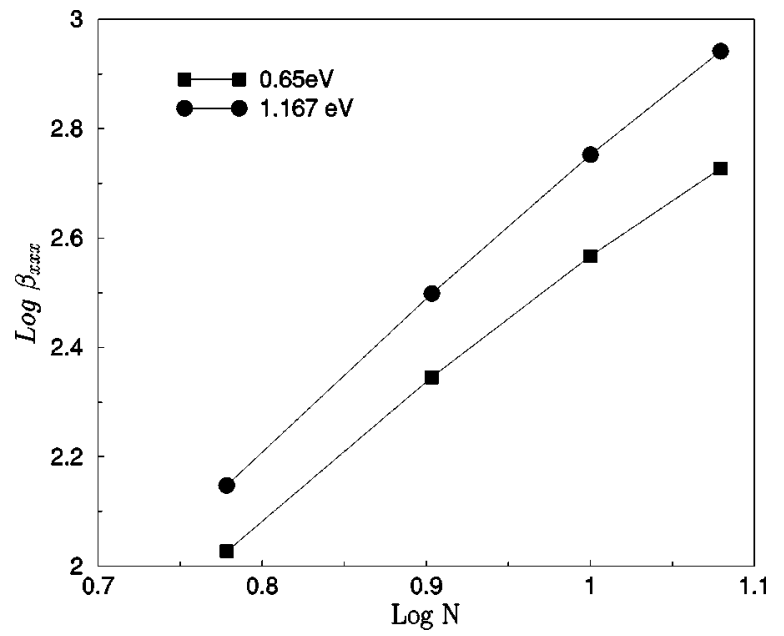

FIG. 3. $\log -\log$ plot of $\beta_{x x x}$ vs system size, $\mathrm{N}$, at two different excitation energies, $0.65 \mathrm{eV}$ and $1.167 \mathrm{eV}$ for push-pull polyenes. terms for $\gamma$ (with $\omega_{\sigma}=\omega_{1}+\omega_{2}+\omega_{3}$ ), respectively. The optic coefficients so computed are exact within the Hilbert space chosen for the Hamiltonian. This is because the correction vectors implicitly incorporate all the excited states of the Hamiltonian in the configuration space in which it is defined. If the Hamiltonian spans a finite dimensional Hilbert space, as in the PPP models, we can get model exact optic coefficients. The linear algebraic equations that result, when Eqs. (3) and (4) are cast in the matrix form, can be solved efficiently by using a small matrix algorithm. ${ }^{30}$

\section{COMPUTATIONAL PROCEDURE}

The parameters for the PPP calculation on carbon and nitrogen systems have been optimized in the past. ${ }^{17,22}$ How-

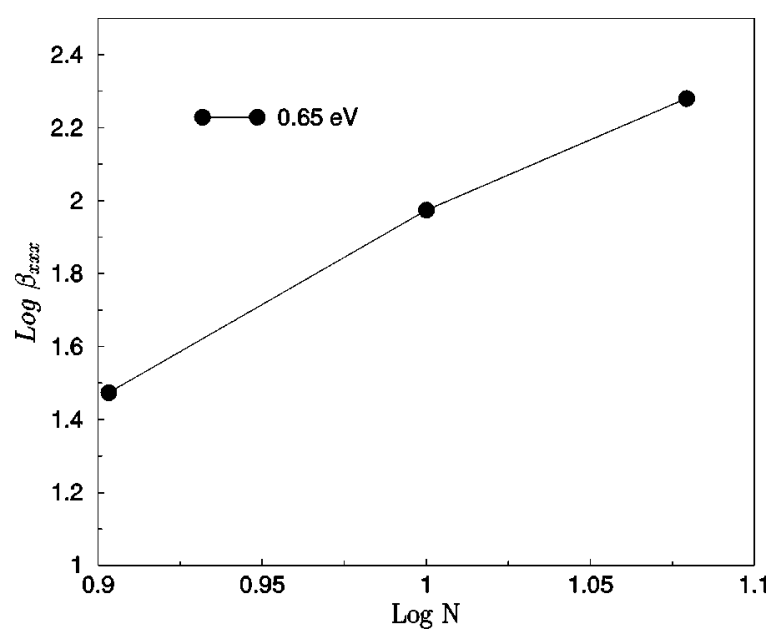

FIG. 4. $\log -\log$ plot of $\beta_{x x x}$ vs system size, $\mathrm{N}$, at $0.65 \mathrm{eV}$ for the unsubstituted $(\mathrm{PN})_{x}$ chains. 


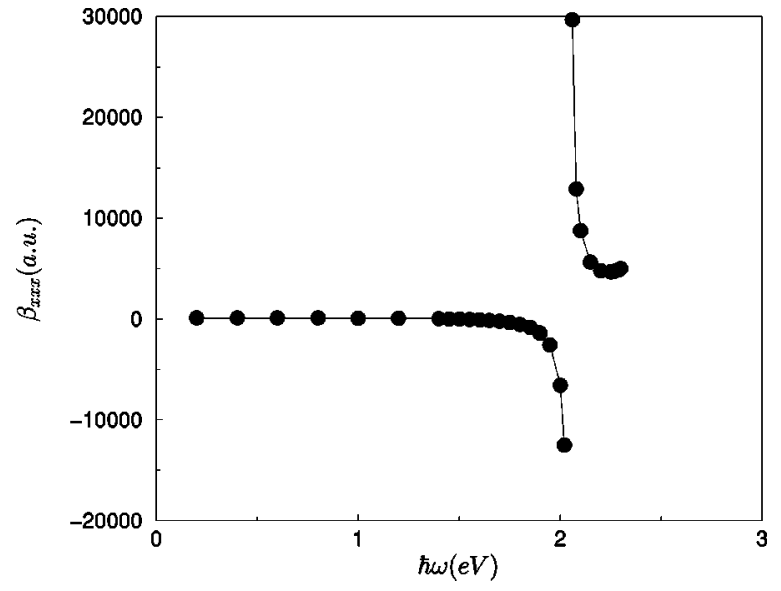

FIG. 5. Dispersion plot of the $(\mathrm{PN})_{5}$ chain for $\beta_{x x x}$.

ever, standard PPP parameters for phosphorus is not known. Therefore, before embarking on NLO calculations on linear phosphazenes, parameterization for phosphorus had to be carried out. The parameters required are: transfer integral for the $\mathrm{P}-\mathrm{N}$ bonds, $t$, the on-site correlation energy, $U_{\mathrm{P}}$ for phosphorus, the $\mathrm{P}-\mathrm{N}$ single and double bond lengths and the site or orbital energies $\epsilon_{P}$ for phosphorus relative to nitrogen. To determine the PPP parameters for phosphorus, we need electronic spectra and the associated optical data on a well defined system consisting of phosphorus and nitrogen atoms in conjugation. The cyclic trimer $(\mathrm{PN})_{3}$ is such a system whose electronic spectra has been studied in some detail. The system has an experimentally determined uniform phosphorusnitrogen bond lengths of $1.62 \AA{ }^{31,32}$ The on-site correlation energy were taken as $11.64 \mathrm{eV}$ for $\mathrm{P}$ and $14.12 \mathrm{eV}$ for $\mathrm{N}$ as suggested by Flurry. ${ }^{33}$ The lower on-site correlation energy for phosphorus compared to that for nitrogen reflects the more diffused nature of the valence orbital in the former. The parameters that were varied were the transfer integral of the $\mathrm{N}-\mathrm{P}$ bond and the site energies on the nitrogen and phosphorus atoms. The site energy $\epsilon_{p}$ on $\mathrm{P}$ is taken as zero and provides a reference for the site energy of nitrogen. Since phosphorus is more electropositive than nitrogen, the nitro-

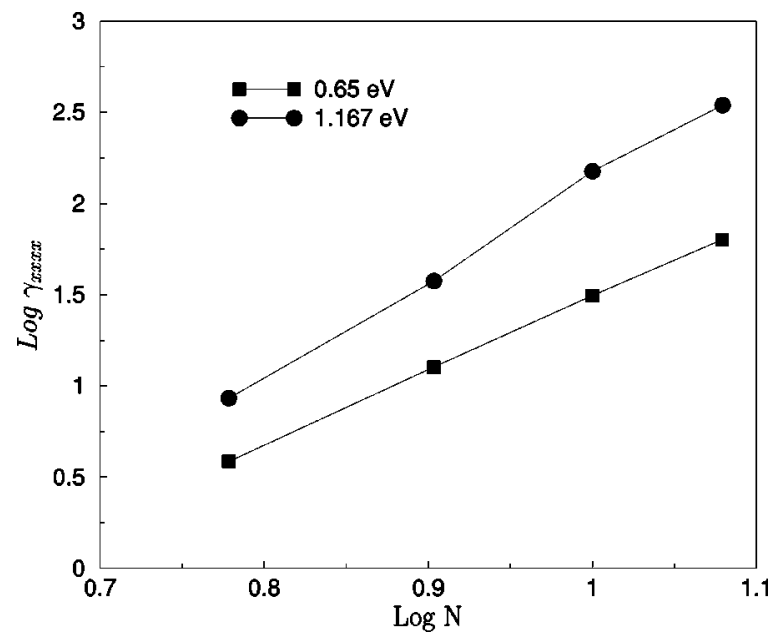

FIG. 6. $\log -\log$ plot of $\gamma_{x x x x}$ vs system size, $\mathrm{N}$, at two different frequencies for push-pull polyenes.

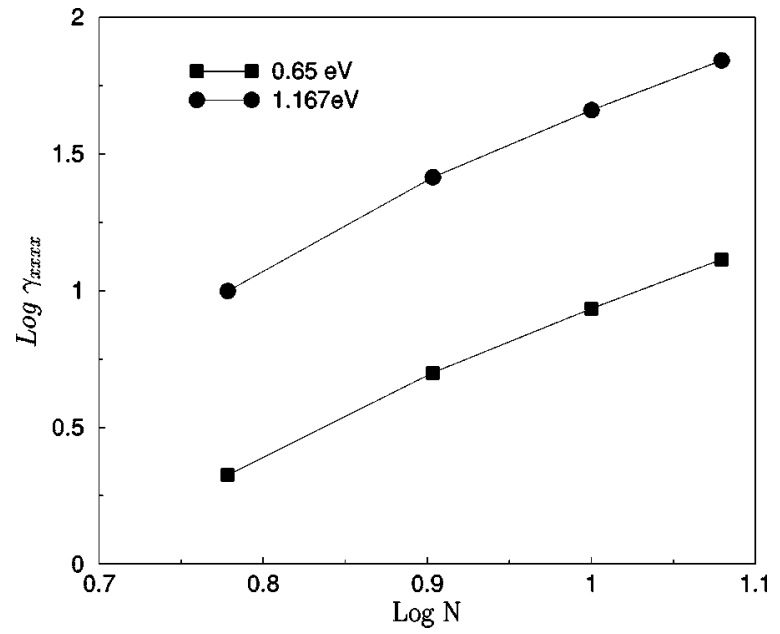

FIG. 7. $\log -\log$ plot of $\gamma_{x x x x}$ vs system size, $\mathrm{N}$, at two different frequencies for push-pull $(\mathrm{PN})_{x}$ systems.

gen site energy is allowed to take only negative values. The transfer integral was varied between 1.6 and $2.0 \mathrm{eV}$.

Properties of all the states were obtained for each parameter set and they included dipole moments, transition dipole moments amongst the low-lying states. The optical gaps $\left(E_{g}\right)$ defined as the energy gap from the ground state to the state to which the transition dipole is appreciable, were obtained for comparison with experiments. The set of parameters which reproduced the best optical gap, when compared with experiments $^{34,35}$ was chosen for all further calculations. The reported absorption spectrum of tricyclophosphazene has an absorption maxima at $217 \mathrm{~nm}$. One set of parameters produced doubly degenerate excited states at $218 \mathrm{~nm}$ with the largest magnitude of the transition dipole moment from the ground state to any of the excited states. There is another doubly degenerate excited state at $198 \mathrm{~nm}$ whose intensity is $\sim 70 \%$ of the intensity of the transition at $217 \mathrm{~nm}$. This, however has not been reported in the literature since most UV-visible measurements usually stop at about $200 \mathrm{~nm}$. The parameters which produced the above excitation gaps in agreement with experiment are $\epsilon_{N}=-5.8 \mathrm{eV}$ and $\epsilon_{P}=0.0$ $\mathrm{eV}$ for the nitrogen and phosphorus atoms respectively. The

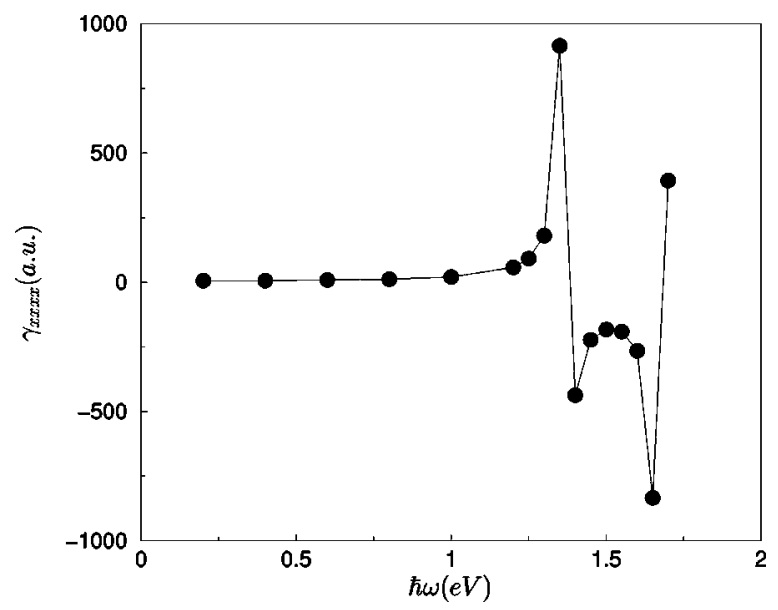

FIG. 8. Dispersion plot of $(\mathrm{PN})_{5}$ for $\gamma_{x x x x}$. 
TABLE VI. Tumbling averaged SHG coefficients (in a.u., 1 a.u. $=8.637 \times 10^{-32}$ esu) and THG coefficients (in $10^{3}$ a.u., 1 a.u. $=5.037 \times 10^{-40}$ esu) of push-pull phosphazenes and push-pull polyenes. A corresponds to the electron pushing group at the $\mathrm{P}$ terminal and electron pulling group at the $\mathrm{N}$ terminal. $\mathrm{B}$ corresponds to the electron pulling group at the $\mathrm{P}$ terminal and electron pushing group at the $\mathrm{N}$ terminal. $\mathrm{C}$ corresponds to push-pull polyenes. In all cases the magnitude of push (pull) corresponds to the site energy of $-1(+1) \mathrm{eV}$, relative to unsubstituted system. At every system size, the numbers in the first line correspond to an excitation energy of $0.65 \mathrm{eV}$ and those on the second line correspond to an excitation energy of $1.167 \mathrm{eV}$.

\begin{tabular}{|c|c|c|c|c|c|c|c|c|c|}
\hline \multirow[b]{2}{*}{$\mathrm{N}$} & \multicolumn{3}{|c|}{ A } & \multicolumn{3}{|c|}{ B } & \multicolumn{3}{|c|}{$\mathrm{C}$} \\
\hline & $\beta_{x}^{\mathrm{av}}$ & $\beta_{y}^{\mathrm{av}}$ & $\gamma^{\mathrm{av}}$ & $\beta_{x}^{\mathrm{av}}$ & $\beta_{y}^{\mathrm{av}}$ & $\gamma^{\mathrm{av}}$ & $\beta_{x}^{\mathrm{av}}$ & $\beta_{y}^{\mathrm{av}}$ & $\gamma^{\mathrm{av}}$ \\
\hline \multirow[t]{2}{*}{6} & -8.159 & 45.93 & 0.7360 & -18.58 & -41.89 & 0.0212 & 113.7 & 13.07 & 0.8861 \\
\hline & $(-67.27)$ & $(52.66)$ & (7.201) & $(-32.98)$ & $(-52.40)$ & $(0.3907)$ & $(149.9)$ & $(19.02)$ & $(1.828)$ \\
\hline \multirow[t]{2}{*}{8} & -2.199 & 33.09 & 1.381 & 16.40 & -68.90 & 0.3404 & 232.0 & 19.36 & 2.952 \\
\hline & $(-90.37)$ & $(32.26)$ & $(14.81)$ & $(5.365)$ & $(-34.79)$ & $(1.855)$ & $(329.5)$ & $(30.81)$ & $(7.931)$ \\
\hline \multirow[t]{2}{*}{10} & 46.12 & 9.084 & 1.908 & 81.39 & -101.9 & 0.8759 & 382.1 & 26.08 & 7.182 \\
\hline & $(-35.95)$ & $(-0.3316)$ & (17.93) & $(88.30)$ & $(-130.9)$ & $(4.559)$ & $(583.4)$ & $(44.65)$ & $(24.92)$ \\
\hline \multirow[t]{2}{*}{12} & -125.19 & 21.74 & 2.474 & 173.1 & -139.1 & 1.611 & 548.4 & 33.08 & 14.34 \\
\hline & $(-72.24)$ & $(40.20)$ & $(20.28)$ & $(216.5)$ & $(-179.1)$ & $(8.632)$ & $(896.2)$ & $(59.97)$ & $(71.41)$ \\
\hline
\end{tabular}

transfer integral is $t_{\mathrm{PN}}=1.76 \mathrm{eV}$. As mentioned earlier, the on-site repulsion parameters were held fixed at the values suggested by Flurry. ${ }^{33}$ The difference in the values of these parameters from the nitrogen atoms in cyanine dyes is due to the fact that we have used phosphorus as the reference atom while in cyanine dyes, carbon was the reference atom. Some differences could also arise due to the qualitative difference that exists between the cyanines and phosphazenes. To obtain transfer integrals for values of bond length different from $1.62 \AA$, we have assumed linear variation of the transfer integral with bond length for small changes in bond length around $1.62 \AA$. Shorter bond lengths correspond to proportionately higher transfer integrals. In case of the $(-\mathrm{P}=\mathrm{N}-)_{x}$ linear chains, the $\mathrm{P}-\mathrm{N}$ single bond length was taken to be $1.74 \AA$ and the $\mathrm{P}=\mathrm{N}$ double bond length was fixed at $1.52 \AA$ corresponding to a $7 \%$ bond length alternation in linear phosphazenes reported previously. ${ }^{36}$ Similar to what was done in the case of polyenes, ${ }^{37}$ the transfer integrals for the $\mathrm{P}-\mathrm{N}$ single and double bonds were fixed at 1.40 and $2.12 \mathrm{eV}$, respectively, assuming that $t_{\mathrm{P}-\mathrm{N}}(r)=t_{\mathrm{P}-\mathrm{N}}\left(r_{0}\right)-3.27\left(r-r_{0}\right)$, where $t_{\mathrm{P}-\mathrm{N}}\left(r_{0}\right)=1.76 \mathrm{eV}$ at $r_{0}=1.62 \AA$. The $\mathrm{P}-\mathrm{N}-\mathrm{P}$ bond angles were fixed at $120^{\circ}$ and the trans geometry was maintained throughout the calculations. The quantities computed include the NLO coefficients $\alpha, \beta, \gamma$ along with the ground state dipole moment and the optical gap. In our calculation, the molecules were considered two dimensional and were confined to the $x-y$ plane. The $z$-components of all the NLO coefficients were, therefore zero. The tumbling average linear polarizability and first and second hyperpolarizabilities are given by

$$
\begin{aligned}
& \bar{\alpha}=(1 / 3) \sum_{i=1}^{3} \alpha_{i i}, \\
& \bar{\beta}_{i}=(1 / 3) \sum_{j=1}^{3}\left(\beta_{i j j}+\beta_{j j i}+\beta_{j i j}\right), \\
& \bar{\gamma}=(1 / 15) \sum_{i=1}^{3} \sum_{j=1}^{3}\left(2 \gamma_{i i j j}+\gamma_{i j j i}\right),
\end{aligned}
$$

which are useful for comparison with experimental results obtained from solution measurements.

\section{RESULTS AND DISCUSSION}

Calculations were performed on a number of planar phosphazene oligomers. Optical gaps, $E_{g}$ (defined as the lowest excited state to which the transition dipole from the ground state is appreciable), dipole moments, and oscillator strengths (calculated from exact transition dipole moments and optical gaps) were obtained for the linear chain phosphazenes, $\mathrm{N}-(\mathrm{P}=\mathrm{N})_{n}-\mathrm{P}$, and for the corresponding cyclic phosphazenes for $n$ varying from 2 to 5 . The variation of the optical gap with inverse system size is shown in Fig. 1. We find that the chains show a very small variation with the system size while the rings show a sharp drop in the optical gap with increase in the system size. Both the systems, however, show a very good linear dependence on inverse system size. This allows for extrapolation of the optical gap to the infinite system size. The extrapolated optical gap obtained from the chains data is $3.89 \mathrm{eV}$ while that obtained from the rings data is $3.51 \mathrm{eV}$. These two values imply that the long chain phosphazenes will have an optical gap of $3.70 \pm 0.19$ $\mathrm{eV}$. This optical gap of $3.7 \mathrm{eV}$ in phosphazenes is much larger than that in polyenes which is $2.8 \mathrm{eV}^{8}$

The ground state dipole moment as well as the transition dipole moment to the lowest excitation increase with increasing $n$. We show a plot of the magnitude of the transition dipole moment vector per PN unit as a function of system size in Fig. 2. Also shown on the same plot is the Hückel transition dipole per PN unit. We note that the Hückel transition dipole is smaller than the transition dipole in the correlated model. This behavior is opposite to what is observed in polyenes where the Hückel theory predicts larger transition dipoles and thus higher intensities. This can be attributed to the fact that the correlations reduce the energy difference between the ionic and the covalent VB configurations in linear phosphazenes. This allows easier charge separation compared to the Hückel model in which the difference in energy between ionic and covalent states is rather large. The Hückel transition dipoles show a weaker dependence on the chain length compared to the transition dipoles in the PPP model (Fig. 2), which can be understood from the fact that Hückel model favors charge separation to be spread out over the entire system compared to the PPP model. 
We have computed the linear and nonlinear optic response coefficients for the $(\mathrm{PN})_{x}$ system and have compared them with those of polyenes and push-pull polyenes (see Tables I and II for the all the components of $\alpha$ ). We find that the $\alpha_{i j}$ values for the $(\mathrm{PN})_{x}$ and $(\mathrm{CH})_{x}$ are comparable in both the unsubstituted and push-pull substituted systems of the same size. The $\alpha_{i j}$ values for the two systems are also comparable at both the frequencies at which we have computed the polarizability.

The second harmonic generation ( $\mathrm{SHG}$ ) coefficients for the $(\mathrm{PN})_{x}$ systems are very different from those of the $(\mathrm{CH})_{x}$ systems. The SHG coefficients are all zero in the latter, in the absence of any substitution, because of the presence of an inversion center. The inversion symmetry can be broken by introducing push-pull groups. In order to compare the pushpull polyenes with the $(\mathrm{PN})_{x}$ system, we have studied the push-pull substituted $(\mathrm{PN})_{x}$ systems.

In Tables III, IV, and $\mathrm{V}$, we present the results for all the nonzero $\beta$ components for the $(\mathrm{PN})_{x}$, push-pull $(\mathrm{PN})_{x}$, and push-pull $(\mathrm{CH})_{x}$ systems at two frequencies, 0.65 and 1.167 $\mathrm{eV}$, the latter being the fundamental frequency of the $\mathrm{Nd}$ YAG laser at which experimental measurements are normally carried out. We have reported unique $\beta$ components since the overall permutation symmetry including the Kleinmann symmetry is valid at these frequencies which are away from resonances. We have also reported the corresponding $\beta$ values from a two level approximation ${ }^{9,38}$ in all these systems. The two levels, we consider, are the ground state and the excited state to which the transition dipole moment has the largest value. This criterion is important since in the $(\mathrm{PN})_{x}$ systems as well as the push-pull polyenes, the transition to the lowest excited singlet is usually weakly allowed as this excited state is derived from a dipole forbidden covalent state of the corresponding system with electron-hole symmetry. We first note that the $\beta$ values calculated from the two-level approximation are in poor agreement with the exact values. Even in the context of multiply substituted pushpull polyenes, it was shown earlier ${ }^{28}$ that the two-level approximation breaks down completely. In the $(\mathrm{PN})_{x}$ systems, each component of the ground state dipole moment is larger than the corresponding component of the dipole moment in the excited state that has large transition dipole to the ground state. This necessarily implies that the low-frequency $\beta$ values are all negative.

The exact $\beta$ value corresponding to the dominant component $\left(\beta_{x x x}\right)$, on the other hand, is positive at the lower excitation energy of $0.65 \mathrm{eV}$ for all the systems and is negative for small system sizes at the higher excitation energy of $1.167 \mathrm{eV}$. The low-frequency $\beta_{x x x}$ values increase superlinearly with system size. The dependence has the form $\beta_{x x x}$ $\sim N^{a}$. It is not possible to find such a dependence at the higher frequency where, depending on the system size, the chain could be on either side of a resonance. Introduction of the push-pull groups in the $(\mathrm{PN})_{x}$ system leads to negative value for $\beta_{x x x}$ component even at the low excitation frequency of $0.65 \mathrm{eV}$ in the $(\mathrm{PN})_{3}$ and $(\mathrm{PN})_{4}$ systems, while it becomes positive for the $(\mathrm{PN})_{5}$ system. Thus, power law for the system size dependence of $\beta_{x x x}$ for the push-pull system cannot be obtained. The push-pull polyenes with site ener- gies corresponding to the push and the pull groups of $\epsilon$ $= \pm 1.0 \mathrm{eV}$ have much larger first hyperpolarizability responses than the $(\mathrm{PN})_{x}$ systems with or without push-pull groups. We also find that the dominant component $\beta_{x x x}$ has the same sign in the push-pull polyenes for all system sizes at both the excitation frequencies. The power law exponent for the push-pull polyenes at $0.65 \mathrm{eV}$ is 2.33 (Fig. 3). In case of the $(\mathrm{PN})_{x}$ system, the $\log -\log$ plot is quite nonlinear. However, taking into account the system sizes corresponding to $(\mathrm{PN})_{4},(\mathrm{PN})_{5}$, and $(\mathrm{PN})_{6}$ we can estimate the power law exponent. We find that in the $(\mathrm{PN})_{x}$ system, in the absence of any push-pull group the exponent at the same excitation frequency is 4.58 (Fig. 4). This shows that the $(\mathrm{PN})_{x}$ system has a stronger dependence on system size for sizes away from the thermodynamic limit. At the thermodynamic limit, the properties become extensive and we should expect a linear dependence of all these coefficients as a function of system size. We have also shown the dispersion of the $\beta_{x x x}$ component as a function of the excitation frequency in Fig. 5. It is interesting to note that the sign of the coefficient $\beta_{x x x}$ changes well before the first singularity. This is because, in the $(\mathrm{PN})_{x}$ system, the sign of all the dipole matrix elements is not the same and the sign of the coefficients cannot be predicted a priori. The first singularity occurs at $2.04 \mathrm{eV}$ in the $(\mathrm{PN})_{5}$ system and corresponds to the excited state at $4.08 \mathrm{eV}$.

The largest component of the THG coefficient of the $(\mathrm{PN})_{x}$ system is the $x x x x$ component and all other components are smaller by over a factor of 5 for the lower excitation frequency $(0.65 \mathrm{eV})$ and by a factor of 20 for the higher excitation frequency $(1.167 \mathrm{eV})$. The dominant component increases by a factor of about 5 in going from the low frequency to the high frequency for all system sizes from $(\mathrm{PN})_{3}$ to $(\mathrm{PN})_{6}$. All the components except the $x x x x$ component are negative at the low excitation frequency. At the higher excitation frequency, the $y x x x$ component also becomes positive. For all system sizes, the $\gamma_{x x x x}$ values of the $(\mathrm{PN})_{x}$ are consistently smaller than the $\gamma_{x x x x}$ values of the corresponding $(\mathrm{CH})_{x}$ systems. At the excitation energy of $0.65 \mathrm{eV}$, the $\gamma_{x x x x}$ values of the $(\mathrm{PN})_{x}$ is smaller by a factor of 2 for hexatrienes and a factor of 6 for the dodecahexenes.

The power law exponent for the major component at the two frequencies are 2.61 at $0.65 \mathrm{eV}$ and 2.79 at $1.167 \mathrm{eV}$ (Fig. 6). We can compare these with the values for the polyenes systems which are 4.04 and 5.43 (Fig. 7) at these frequencies, respectively. This implies that the THG coefficients would be far smaller for $(\mathrm{PN})_{x}$ systems than for the polyene systems of the same size at higher chain lengths. We also show in Fig. 8 the dispersion relation for the largest component $\gamma_{x x x x}$. Thus, while the $(\mathrm{PN})_{x}$ systems have nonzero SHG response, they are not as good as polyenes for THG.

We have also studied push-pull $(\mathrm{PN})_{x}$ systems by introducing an electron pushing group at the terminal $\mathrm{P}$ site and an electron pulling group at the terminal $\mathrm{N}$ site. The behavior of the dominant components of $\beta$ in the push-pull systems as a function of size do not show any regular trend. At 0.65 $\mathrm{eV}$ excitation frequency, the $\beta_{x x x}$ component has a negative sign for the $(\mathrm{PN})_{3},(\mathrm{PN})_{4}$, and $(\mathrm{PN})_{6}$ systems but the sign is positive for the $(\mathrm{PN})_{5}$ system. Even among the first three oligomers, the magnitude changes nonmonotonically. The 
$\beta_{x x y}$ component in the two shorter oligomers are positive and larger than the $\beta_{x x x}$ components. At $1.167 \mathrm{eV}$ excitation frequency, for all the oligomers, $\beta_{x x x}$ values have the same negative sign but the variation is nonmonotonic. Introducing an electron pulling group at the phosphorus end seems to improve the SHG response of the system, when compared to the response with electron pushing group at the phosphorus end. In Table VI, we present the tumbling averaged $\beta$ values for various system sizes for the two kinds of substitutions. We see that the magnitude of the SHG response in all cases is larger with electron pulling group at the phosphorus end and electron pushing group at the nitrogen end. However, as we can see from Table VI, the SHG response of substituted push-pull systems is much smaller than in the corresponding push-pull polyene systems.

The THG coefficients show a marginal increase with introduction of the push-pull groups, if the electron pushing group is at the phosphorus end (Table VI). The THG response becomes weaker if the pushing and pulling group positions are reversed. In both cases, the tumbling averaged THG coefficients are smaller than in the corresponding push-pull polyenes (Table VI). The power law exponent for the dominant component with electron pushing group at the phosphorus end, at $0.65 \mathrm{eV}$ is 1.8 , which is smaller than the value for the unsubstituted $(\mathrm{PN})_{x}$ system at the same excitation frequency. Even at $1.167 \mathrm{eV}$ excitation frequency, the dominant THG component, $\gamma_{x x x x}$, shows only a slight monotonic increase. The power law exponent at this frequency is smaller at 1.5. This shows that introducing pushpull groups is not conducive for increasing the SHG or the THG response of the $(\mathrm{PN})_{x}$ systems.

\section{CONCLUSION}

In this paper, we have investigated the linear and nonlinear optical properties of the inorganic analogs of the polyenes, namely $(\mathrm{PN})_{x}$ systems, theoretically using the PariserParr-Pople model. We have fixed the nitrogen and phosphorus parameters for the PPP model by fitting the lowlying spectrum known experimentally in a few systems. The optical gap in the thermodynamic limit of the $(\mathrm{PN})_{x}$ system is much larger than that in the polyenes. We have compared their low-lying electronic excitation and dynamic linear and nonlinear optic responses with those of polyenes. The $(\mathrm{PN})_{x}$ systems exhibit linear and THG responses comparable with those of polyenes. However, they exhibit weaker SHG response than the push-pull polyenes. This could be because $(\mathrm{PN})_{x}$ system can be viewed as a polyene system with alternate push and pull groups introduced along the chain atoms. In the case of polyenes, such a substitution pattern has been shown to be less efficient for SHG than when the push and pull groups are introduced only on the terminal sites of the chain. ${ }^{28}$ These coefficients also have a weaker system size dependence than that in the polyenes. If push-pull groups are introduced in the linear chain phosphazenes, then a pushing group at the P-terminal end and a pulling group at the $\mathrm{N}$-terminal end will be desirable.

\section{ACKNOWLEDGMENT}

P.K.D. and S.R. thank the Council for Scientific and Industrial Research, Government of India for funding.

${ }^{1}$ S. R. Marder, J. E. Sohn, and G. D. Stucky, Materials for Nonlinear Optics, ACS Symp. Ser. 455 (American Chemical Society, Washington, D.C., 1991).

${ }^{2}$ R. G. Denning, J. Mater. Chem. 11, 19 (2001).

${ }^{3}$ S. R. Marder, Lap-Tak Cheng, B. G. Tiemann, A. C. Friedli, M. Blanchard-Desce, J. W. Perry, and J. Skindhoj, Science 263, 511 (1994).

${ }^{4}$ H. Ikeda, Y. Kawabe, and T. Kawasaki, Chem. Phys. Lett. 157, 576 (1989).

${ }^{5}$ L.-T. Cheng, W. Tam, S. R. Marder, A. E. Stiegman, G. Rikken, and C. W. Spangler, J. Phys. Chem. 95, 10643 (1991).

${ }^{6}$ J. O. Morley, P. Pavlides, and D. Pugh, J. Chem. Soc., Faraday Trans. 2 85, 1789 (1989).

${ }^{7}$ Z. G. Soos and S. Ramasesha, J. Chem. Phys. 90, 1067 (1989).

${ }^{8}$ I. D. L. Albert, J. O. Morley, and D. Pugh, J. Chem. Phys. 99, 5197 (1993).

${ }^{9}$ S. Ramasesha and P. K. Das, Chem. Phys. 145, 343 (1990).

${ }^{10}$ A. E. Stiegman, E. Graham, K. J. Perry, L. R. Khundkar, L. T. Cheng, and J. W. Perry, J. Am. Chem. Soc. 113, 7658 (1991).

${ }^{11}$ I. D. L. Albert, J. O. Morley, and D. Pugh, J. Phys. Chem. A 101, 1763 (1997)

${ }^{12}$ I. D. L. Albert, D. Pugh, J. O. Morley, and S. Ramasesha, J. Phys. Chem. 96, 10160 (1992).

${ }^{13}$ S. H. Stevenson, D. S. Donald, and G. R. Meredith, Mater. Res. Soc. Symp. Proc. 109, 103 (1988).

${ }^{14}$ B. M. Pierce, Proc. SPIE 1560, 1 (1991).

${ }^{15}$ M. Blanchard-Desce, C. Runser, A. Fort, M. Barzoukas, J. M. Lehn, V. Bloy, and V. Alain, Chem. Phys. 199, 253 (1995).

${ }^{16}$ R. A. Huijts and G. L. J. Hesselink, Chem. Phys. Lett. 156, 209 (1989).

${ }^{17}$ I. D. L. Albert, "A theoretical study of the linear and nonlinear optical properties of conjugated systems," Ph.D. thesis, 1991.

${ }^{18}$ R. Pariser and R. G. Parr, J. Chem. Phys. 21, 466 (1953); J. A. Pople, Trans. Faraday Soc. 49, 1375 (1953).

${ }^{19}$ N. L. Paddock, Q. Rev., Chem. Soc. 18, 168 (1964).

${ }^{20}$ M. J. S. Dewar, E. A. C. Lucken, and M. A. Whitehead, J. Chem. Phys. 1960, 2423.

${ }^{21}$ J. E. Huheey, E. A. Keiter, and R. L. Keiter, Inorganic Chemistry: Principles of Structure and Reactivity, 4th ed. (Addison-Wesley, New York, 1993), p. 770.

${ }^{22}$ Z. G. Soos and S. Ramasesha, Phys. Rev. B 29, 5410 (1984).

${ }^{23}$ S. Ramasesha, Z. Shuai, and J. L. Bredas, Chem. Phys. Lett. 245, 224 (1995).

${ }^{24}$ K. Ohno, Theor. Chim. Acta 2, 219 (1964).

${ }^{25}$ S. Ramasesha, Swapan K. Pati, Z. Shuai, and J. L. Bredas, Adv. Quantum Chem. 38, 121 (2000).

${ }^{26}$ S. R. Marder, C. B. Gorman, B. G. Tiemann, and Lap-Tak Cheng, J. Am. Chem. Soc. 115, 3006 (1993)

${ }^{27}$ I. D. L. Albert, J. O. Morley, and D. Pugh, J. Chem. Phys. 102, 237 (1995).

${ }^{28}$ I. D. L. Albert, P. K. Das, and S. Ramasesha, Chem. Phys. Lett. 168, 454 (1990).

${ }^{29}$ V. J. Docherty, D. Pugh, and J. O. Morley, J. Chem. Soc. Faraday Trans. 81, 1179 (1985).

${ }^{30}$ S. Ramasesha, J. Comput. Chem. 11, 545 (1990).

${ }^{31}$ R. Keat, R. Soc. Chem. London 6, 182 (1974); 7, 188 (1977); 8, 204 (1979); 9, 210 (1979); 10, 232 (1979); 11, 219 (1980).

${ }^{32}$ J. C. Clardy, R. L. Kolpa, and J. G. Verkade, Phosphorus 4, 133 (1974).

${ }^{33}$ R. L. Flurry, Molecular Orbital Theories of Bonding in Organic Molecules (Marcel Dekker, New York. 1968).

${ }^{34}$ B. Lakatos, A. Hesz, Zs. Vetessy, and G. Horvath, Acta Chim. Acad. Sci. Hung. 60, 309 (1969).

${ }^{35}$ N. B. Jurinski, C. C. Thompson, and P. A. D. De Maine, J. Inorg. Nucl. Chem. 27, 1571 (1965).

${ }^{36}$ H. Sun, J. Am. Chem. Soc. 119, 3611 (1997).

${ }^{37}$ L. R. Ducasse, T. E. Miller, and Z. G. Soos, J. Chem. Phys. 76, 4094 (1982).

${ }^{38}$ J. L. Oudar and D. S. Chemla, J. Chem. Phys. 66, 2664 (1977). 